\title{
The perils of anthropomorphism
}

\section{Consciousness should be ascribed to animals only with extreme caution.}

\section{Clive D. L. Wynne}

$\mathrm{T}$ he complexity of animal behaviour naturally prompts us to use terms that are familiar from everyday descriptions of our own actions. Charles Darwin used mentalistic terms freely when describing, for example, pleasure and disappointment in dogs; the cunning of a cobra; and sympathy in crows. Darwin's careful anthropomorphism, when combined with meticulous description, provided a scientific basis for obvious resemblances between the behaviour and psychology of humans and other animals. It raised few objections.

The 1890s saw a strong reaction against ascribing conscious thoughts to animals. In the United Kingdom, Conwy Lloyd Morgan's canon forbade the explanation of animal behaviour with "a higher psychical faculty" than demanded by the data. In the United States, Edward Thorndike advocated replacing the use of anecdotes in the study of animal behaviour with controlled experiments. $\mathrm{He}$ argued that when studied in controlled and reproducible environments, animal behaviour revealed simple mechanical laws that made mentalistic explanations unnecessary.

This rejection of anthropomorphism was one of the few founding principles of behaviourism that survived the rise of ethological and cognitive approaches to studying animal behaviour. But after a century of silence, recent decades have seen a resurgence of anthropomorphism.

This movement was led by ethologist Donald Griffin, famous for his discovery of bat sonar. Griffin argued that the complexity of animal behaviour implies conscious beliefs and desires, and that an anthropomorphic explanation can be more parsimonious than one built solely on behavioural laws. Griffin postulated, "Insofar as animals have conscious experiences, this is a significant fact about their nature and their lives." Animal communication particularly impressed Griffin as implying animal consciousness.

Griffin has inspired several researchers to develop ways of making anthropomorphism into a constructive tool for understanding animal behaviour. Gordon Burghardt was keen to distinguish the impulse that prompts children to engage in conversations with the family dog (naive anthropomorphism) from 'critical anthropomorphism', which uses the assumption of animal consciousness as a "heuristic method to formulate research agendas that result in publicly verifiable data that move our understanding of behaviour forward." Burghardt points to the deathfeigning behaviour of snakes and possums as

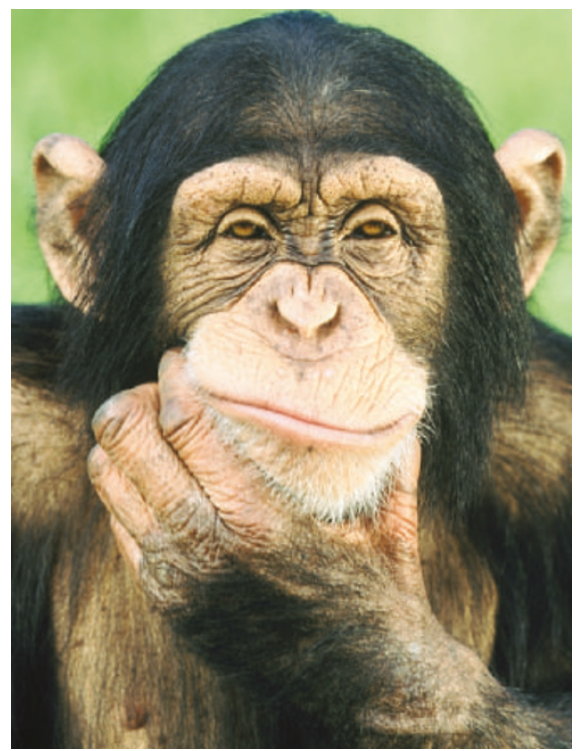

Lost in thought: but do animals think like we do?

examples of complex and apparently deceitful behaviours that can best be understood by assuming that animals have conscious states.

Burghardt's critical and naive anthropomorphisms are akin to Frans de Waal's distinction between 'animal-centred' and 'anthropocentric' anthropomorphism. For de Waal, denying human-like thought processes in cases where its assumption is warranted is just as dangerous as to anthropomorphize inappropriately. So seriously does de Waal view this error that he terms it 'anthropodenial' - "a blindness to the human-like characteristics of animals, or the animal-like characteristics of ourselves". De Waal proposed his research on reconciliation in chimpanzees as an example of the practical utility of anthropomorphism. Earlier studies had viewed aggression within a group as a means of maintaining space between individuals - which predicts reduced contact between individuals after aggressive encounters. De Waal used anthropomorphism to predict that the need for social cohesion would lead to reconciliation after conflicts - this was confirmed by an increased rate of affiliative contact between combatants after aggression.

But anthropomorphism is not a welldeveloped scientific system. On the contrary, its hypotheses are generally nothing more than informal folk psychology, and may be of no more use to the scientific psychologist than folk physics to a trained physicist. Although anthropomorphism may on occasion be a source of useful hypotheses about animal behaviour, acknowledging this does not concede the general utility of an anthropomorphic approach to animal behaviour.
John Staddon's model of animal short-term memorylikens the process of forgetting to fluid leaking from buckets. But the utility of this model does not point to any general similarity between animal memory and fluid in buckets.

The philosopher Daniel Dennett argues along with Griffin, Burghardt and de Waal that appeals to mentalistic intentional states are more parsimonious than "an abstemiously behaviouristic story of unimaginable complexity". Certainly, attempts to explain complex behaviour in terms of simple mechanisms can become highly convoluted. But to argue that mentalistic accounts are simpler is, as Mark Blumberg and Ed Wasserman point out, to commit the nominal fallacy to believe that giving something a name is the same as explaining it.

Consider the dance language of honeybees that impressed Griffin as possible evidence of consciousness. Foraging bees communicate the direction, distance and quality of a nectar source to their hive-mates. However, their 'language' is much more limited than human language. Honeybees communicate only three dimensions of experience, and only about two things (nectar sources and potential hive sites). Human communication is, in principle, unlimited in the number of dimensions of experience it can convey. To point out that if people were to communicate about nectar sources, we would do so consciously, offers no clue as to how bees might do it. Analogies at least as useful in understanding honeybee communication can be found in the activity of networked computers (unconsciously) establishing communication protocols.

Old-time behaviourism may have imposed excessive constraints on animal psychology. But the reintroduction of anthropomorphism risks bringing back the dirty bathwater as we rescue the baby. The study of animal behaviour is not so mature that it can thoughtlessly reject analogies from any source. But progress will surely be most rapid when we adopt explanatory frameworks that are concrete and unambiguous. Anthropomorphism, even critical or animal-centred, cannot offer that constructive basis.

Clive D. L. Wynne is in the Department of

Psychology, University of Florida, Gainesville, Florida 32611-2250, USA.

\section{FURTHER READING}

Blumberg, M. S. \& Wasserman, E. A. Am. Psychol. 50, 133-144 (1995).

De Waal, F. B. M. Phil. Top. 27, 255-280 (1999).

Mitchell, R. W. et al. Anthropomorphism, Anecdotes and Animals (State Univ. New York Press, New York, 1997). Wynne, C. D. L. Do Animals Think? (Princeton Univ.

Press, Princeton, New Jersey, 2004). 